\title{
Über die Darstellung kristallisierter Taurocholsäure.
}

\author{
Von
}

Olof Hammarsten.

(Der Redaktion zugegangen am 27. August 1904.)

Während die Darstellung reiner Glykocholsäure leicht gelingt, ist dagegen die Darstellung von reiner, kristallisierter Taurocholsäure mit viel größeren Schwierigkeiten verknüpft. Es ist sogar fraglich, ob die Reindarstellung dieser Säure überhaupt bisher jemandem gelungen war. Meines Wissens ist auch zu dem Zwecke nur eine Methode, nämlich die von Parke herrührende, ausgearbeitet worden. Parke, welcher unter Hoppe-Seyler ${ }^{1}$ ) arbeitete, verwendete entfärbte, kristallisierte Hundegalle, welche der gang und gäben Ansicht nach nur Taurocholsäure enthalten soll. Der in der wässerigen Lösung mit Bleiessig und ein wenig Ammoniak erzeugte Niederschlag wurde gut ausgewaschen und mit heißem absoluten Alkohol ausgekocht. Es wurde dann heiß filtriert, das Filtrat mit Schwefelwasserstoff entbleit und das neue, bei mäßiger Wärme auf ein kleines Volumen verdunstete Filtrat mit großem Überschuß von Äther gefällt. Der sirupartige Niederschlag ging nach einiger Zeit größtenteils in feine, seideglänzende, nadelförmige Kristalle über, nie gelang es aber, die ganze Masse zur Kristallisation $\mathrm{zu}$ bringen. Die so gewonnenen Kristalle zerflossen an der Luft sehr schnell. Auch beim Trocknen in trockener, durchgeleiteter Luft gingen sie in eine durchsichtige, amorphe Masse über, die nicht durch Äther allein, wohl aber beim nachherigen Zusatz von ein paar Tropfen Alkohol wieder zur Kristallisation gebracht werden konnte.

1) Medizinisch-chemische Untersuchungen von Felix HoppeSeyler, Berlin 1866, Heft 1, S. 160. 
Aus dem erhaltenen Produkte konnte Parke sowohl Taurin wie Cholsäure darstellen, und es steht wohl also außer Zweifel, daß die Masse Taurocholsäure enthielt. Beim Verbrennen auf dem Platinbleche hinterließen die Kristalle keine Asche, und das Blech, mit Wasser benetzt, gab keine Trübung mit Chlorbaryumlösung. Offenbar handelte es sich also, wie aus der Darstellungsmethode zu erwarten war, um eine freie Säure, bezw. ein Gemenge von solchen. Inwieweit die Kristalle aus reiner Taurocholsäure oder aus einem Gemenge von solcher mit durch Zersetzung entstandener Cholsäure bestanden haben, ist aber aus dem Aufsatze nicht zu ersehen, denn es ist leider keine Bestimmung des Schwefelgehaltes mitgeteilt worden. Etwas befremdend klingt auch die Angabe, daß die bei niederer Temperatur getrocknete Säure weit über $100^{\circ}$ ohne Zersetzung erhitzt werden konnte, was mit der leichten Zersetzbarkeit der Taurocholsäure schwer zu vereinbaren ist und, wie aus dem folgenden hervorgehen wird, jedenfalls nicht mit dem Verhalten der von mir dargestellten Säure stimmt.

Auf Grund einer Analyse von Bensch, ${ }^{1}$ ) welcher in der Hundegalle 6,21\% Schwefel fand, vermutete schon Strecker, daß die Hundegalle nur Taurocholat enthält. Diese Ansicht fand Hoppe-Seyler ${ }^{2}$ ) durch seine Untersuchungen bestätigt, und seitdem dürfte sie wohl allgemein als anerkannt richtig gelten. Aber selbst wenn die Hundegalle ausschließlich Taurocholat enthält, so folgt daraus noch nicht, daß in ihr nur das gewöhnliche Taurocholat vorkommt. Dies ist allerdings von Hoppe-Seyler auf Grund seiner Untersuchungen behauptet worden, mit meiner Erfahrung stimmt aber eine solche Ansicht nicht. Wie aus dem folgenden ersichtlich sein wird, habe ich nämlich aus der Hundegalle außer der gewöhnlichen Taurocholsäure noch eine zweite Taurocholsäure isolieren können, und aus dem Grunde bleibt es noch mehr fraglich, ob Parke wirklich reine, gewöhnliche Taurocholsäure in den Händen gehabt hat.

1) Annal. d. Chem. u. Pharm., Bd. 65.

2) Journal f. prakt. Chemie, Bd. 89 (1863). 
Wenn aber die Hundegalle zwei Taurocholate enthalten kann, welche beide von Bleiessig gefällt werden, so folgt hieraus, daß die bisher geübte Methode keine zur Reingewinnung der gewöhnlichen Taurocholsäure geeignete Methode sein kann. Hierzu kommt noch, daß die nach dieser Methode gewonnene Säure nur zum Teil kristallisiert und sehr schnell an der Luft zerfließt. Da es nun für meine vergleichenden Untersuchungen von Tiergallen notwendig war, die verschiedenen gepaarten Säuren wenn möglich zu isolieren und rein darzustellen, und da die Darstellung der Taurocholsäure aus Rindergalle durch fraktionierte Fällung mit Bleisalzen sehr schlechte Resultate gibt, habe ich eine neue Methode zur Reindarstellung der Taurocholsäuren und in erster Linie der gewöhnlichen Taurocholsäure ausgearbeitet. Der Ausgangspunkt meiner hierher gehörenden Versuche war die folgende Beobachtung.

Es sind in meinem Laboratorium seit einiger Zeit Untersuchungen über das Verhalten verschiedener Gallen zu Neutralsalzen angestellt worden, und über einen Teil dieser Untersuchungen ist schon von Tengströ $\mathrm{m}^{1}$ ) berichtet worden. Er hatte unter anderem gefunden, daß Seifen, namentlich die Ölseife, das Aussalzen des Glyko- und Taurocholates durch Sättigung mit $\mathrm{NaCl}$ verhindern können. Ich versuchte nun diese Wirkung der Gallenseifen dadurch zu eliminieren, daß ich die mit NaCl gesättigte, nicht aussalzbare, schleimfreie Rindergalle nach Zusatz von etwas Äther mit Salzsäure schwach ansäuerte. Es schieden sich hierbei bisweilen (aber bei weitem nicht immer) nach kürzerer oder längerer Zeit, in einzelnen Fällen sogar binnen einer Stunde, schöne farblose Kristallnadeln aus, deren Menge allmählich bedeutend sich vermehrte. Es handelte sich hierbei um solche Gallen, welche nach Zusatz von Äther und Salzsäure allein ohne NaCl keine Kristalle lieferten, und es war also wahrscheinlich, daß - insoferne hier nicht eine Zersetzung unter. Cholsäureabscheidung stattgefunden hatte - das Sättigen mit NaCl das Auskristallisieren der Gallensäuren wesentlich erleichterte. Ich ging deshalb zu Versuchen mit reinem Taurocholat über, um zu sehen, ob die Taurocholsäure aus

1) Diese Zeitschrift, Bd. XLI.

Hoppe-Seyler's Zeitschrift f. physiol. Chemie. XLIII. 
salzgesättigter Lösung kristallisieren könnte. Diese Vermutung fand ich schon beim ersten Versuche bestätigt.

Da das reine Taurocholat bei Zimmertemperatur schon bei neutraler oder schwach alkalischer Reaktion von $\mathrm{NaCl}$, bis zur Sättigung eingetragen, ausgesalzen wird, war es selbstverständlich hier notwendig, den umgekehrten Weg einzuschlagen, d. h. die Taurocholatlösung erst mit Säure $\mathrm{zu}$ versetzen und darauf mit $\mathrm{NaCl}$ zu sättigen. Als Beispiel führe ich den folgenden Versuch an.

$\mathrm{Zu}$ diesem Versuche verwendete ich reines Taurocholat aus Dorschgalle, welches nach der Ausfällung dieser Galle mit Eisenchloridlösung durch Aussalzen des Filtrates mit $\mathrm{NaCl}$ gewonnen worden war. Das Taurocholat wurde in Wasser gelöst, zum zweitenmal mit $\mathrm{NaCl}$ gefällt und dann durch wiederholte Alkoholbehandlung von $\mathrm{NaCl}$ befreit. Das rein weiße Salz enthielt 5,9\% Schwefel (5,96\% berechnet) und war also reines Taurocholat.

Von diesem Salze wurde eine 2\%ige Lösung in Wasser bereitet, mit $2 \% \mathrm{HCl}$ versetzt und unmittelbar darauf mit feingepulvertem $\mathrm{NaCl}$ gesättigt. Es fand hierbei fast sogleich eine sehr geringfügige Ausfällung von Taurocholat (oder Säure) statt. Unmittelbar nach der Filtration wurde die klare Lösung mit Äther versetzt und umgeschüttelt. Innerhalb ein paar Stunden fingen farblose Nadeln an, sich abzuscheiden; ihre Menge vermehrte sich immer mehr und am folgenden Tage war die ganze Probe in einen dicken Kristallbrei umgewandelt worden. Die Flüssigkeit wurde nun mittels der Luftpumpe abgesaugt und die Kristallmasse zwischen Papier stark gepreßt. In der abgesogenen Flüssigkeit trat bald eine neue Kristallisation auf, die wie die vorige behandelt wurde.

Die gesamte ausgepreßte Kristallmasse wurde in wasserhaltigem Alkohol gelöst und durch Filtration von verunreinigendem $\mathrm{NaCl}$ getrennt. Das alkoholische Filtrat wurde darauf mit Äther bis zu starker, bleibender Opalescenz versetzt und stehen gelassen. Nach einigen Stunden setzten sich Gruppen von feinen Kristallnadeln $a b$, und am folgenden Tage enthielt die Flüssigkeit eine reichliche Kristallisation. Durch wiederholten vorsichtigen $\mathrm{Zu}$ - 
satz von Äther konnten neue Kristallisationen, die ebenfalls ganz frei von amorphen Beimengungen waren, erhalten werden. Die gewonnenen Kristalle, welcke keine Neigung zu Wasseraufnahme und Zerfließen an der Luft zeigten, wurden nun in absolutem Alkohol gelöst, und diese, durch Filtration von einer kleinen Menge verunreinigendem $\mathrm{NaCl}$ getrennte Lösung mit Äther versetzt. Hierbei schied sich die Säure indessen amorph aus, und sie hatte keine Neigung, zu kristallisieren, ebenso wenig wie an der Luft zu zerfließen. Nach dem Abgießen der alkoholisch-ätherischen Lösung und Abspülen der amorphen Masse mit Äther konnte sie leicht im Vacuum getrocknet und dann zerrieben werden. Diese fein zerriebene, amorphe Säure hatte ebenso wenig wie die kristallisierte die von Parke beobachtete Eigenschaft, an der Luft zu zerfließen, und sie wurde sehr leicht im Exsikkator zu konstantem Gewicht getrocknet.

Die Säure löste sich in Alkohol und leicht in Wasser zu einer stark sauer reagierenden Lösung von säuerlichem und überwiegend süßem, an Süßholzextrakt erinnerndem Geschmack. Auf dem Platinbleche verbrannt, hinterließ sie keine wägbare Menge Asche. Es handelte sich also hier offenbar um eine Säure, und da es vor allem darauf ankam, zu ermitteln, ob sie Taurocholsäure war, verwendete ich einen Teil der zweimal mit Äther gefältten, amorphen, zu konstantem Gewicht im Exsikkator getrockneten Säure zu einer Schwefelbestimmung.

$$
\begin{gathered}
0,387 \mathrm{~g} \text { Substanz lieferten } 0,170 \mathrm{~g} \mathrm{BaSO}_{4} \\
=0,023353 \mathrm{~g} \text { Schwefel }=6,03 \% \mathrm{~S} .
\end{gathered}
$$

Der für die freie Taurocholsäure berechnete Gehalt an Schwefel ist 6,21\%, während bei der Analyse 6,03\% S gefunden wurden. Die Säure war also als reine oder fast ganz reine Taurocholsäure anzusehen.

Der Umstand, daß die in absolutem Alkohol gelöste kristallisierte Säure beim Umfällen mit Äther amorph ausfiel, veranlaßte einige besondere Versuche, und ich fand hierbei, was ich später wiederholt bestätigt gefunden habe, daß zur. raschen und schönen Kristallisation die Gegenwart von ein wenig Wasser im Alkohol erforderlich ist. Da ich einen Teil der amorphen Säure in absolutem Alkohol gelöst hatte, fiel 
sie wiederum durch Ätherzusatz amorph aus, fügte ich aber vor dem Ätherzusatz einige Tropfen Wasser hinzu, so erhielt ich binnen kurzem zentimeterlange Nadeln, oder richtiger, sehr schlanke vierseitige Prismen, die ebenfalls luftbeständig waren.

Nach diesen Vorversuchen schien also ein neuer Weg zur Reindarstellung der Taurocholsäure gefunden zu sein. Die fortgesetzten Untersuchungen zeigten aber, daß die neue Methode, namentlich bei Verarbeitung von Gallen anderer Tiere, nicht immer sicher zum Ziele führte. Ich fand nämlich mehrmals, daß die in obiger Weise gewonnene Säure von etwas Taurocholat verunreinigt war. In einigen Fällen fand bei Zusatz von $\mathrm{NaCl} \mathrm{zu}$ der angesäuerten Taurocholatlösung schon vor dem vollständigen Sättigen mit $\mathrm{NaCl}$ eine Ausscheidung von mit Taurocholat gemischter Säure statt und dies wiederholte sich nach jeder neuen Filtration. Wenn aber die Säure einmal von gallensaurem Salz verunreinigt worden ist, wird das letztere bei jeder Umfällung durch Ätherzusatz mit der Säure zusammengefällt.

Aus diesem Grunde habe ich die obige Methode als weniger sicher verlassen, und wenn ich sie trotzdem hier oben beschrieben habe, geschah dies, um die Einwirkung des Kochsalzes auf die Kristallisation der wohl nie trüher aus wässeriger Lösung in Kristallen erhaltenen Taurocholsäure zu zeigen. Ich will deshalb auch in diesem Zusammenhange mitteilen, daß die Taurocholsäure sogar ohne Zusatz von Äther aus einer angesäuerten und darauf mit $\mathrm{NaCl}$ gesättigten Taurocholatlösung (allerdings von ein wenig Taurocholat verunreinigt) kristallisieren kann.

Statt des Säureaussalzungsverfahrens habe ich also später eine andere Methode angewendet, deren Prinzip das folgende ist. Das reine Taurocholat wird in alkoholischer Lösung mit einer Säure, Schwefel- oder Chlorwasserstoffsäure, zersetzt und die vom Sulfat, bezw. Chlorid abfiltrierte klare Lösung mit .überschüssigem Äther gefällt. Durch wiederholte Auflösung in Alkohol und Ausfällung mit Äther wird die Säure gereinigt.

Die Lösung des Taurocholates in Alkohol wird unter Umrühren mit schwefelsäurehaltigem Alkohol, bis zu 2-3\% 
Schwefelsäure, oder mit Salzsäure, bis zu 2\% Chlorwasserstoff, versetzt. Die ausgefällten Salze werden abfiltriert und das Filtrat mit überschüssigem Äther gefällt. Sobald die Säure als flockige oder harzige Masse sich ausgeschieden hat - also ohne die Kristallisation abzuwarten -, wird die Flüssigkeit abgegossen und nötigenfalls, um Verluste zu vermeiden, filtriert. Ich habe allerdings die Säure mehrmals ohne Nachteil 24-48 Stunden mit der sauren Flüssigkeit in Berührung gelassen; der Vorsicht halber dürfte es jedoch besser sein, die ausgefällte Säure sobald als möglich von der Flüssigkeit zu trennen. Die ausgefällte Säure wird nun in wenig absolutem Alkohol gelöst, die Lösung filtriert und von neuem mit Äther amorph gefällt. Die Säure löst sich nun regelmäßig in absolutem Alkohol klar und obne Rückstand (Sulfate, Chloride) auf; wenn dies nicht der Fall sein sollte, muß die obige Prozedur selbstverständlich wiederholt werden. Dem alkoholischen Filtrate setzt man ein wenig Wasser hinzu und versetzt darauf mit Äther bis zu starker Opaleszenz oder schwach milchiger Trübung. Wenn hierbei eine geringe amorphe Fällung entstehen würde, wird sie möglichst rasch abfiltriert und man fügt dann, wenn nötig, noch ein wenig Äther hinzu. Nach einiger Zeit fängt die Kristallisation der Säure an, und wenn die Menge der Kristalle sich nicht weiter vermehrt, fügt man der klaren Flüssigkeit wieder Äther bis zur schwach milchigen Trübung hinzu. Diese Prozedur wiederholt man, bis keine beachtenswerte weitere Kristallisation geschieht, und nach demselben Prinzip kann dann die Säure beliebig oft aus schwächerem Alkohol umkristallisiert werden.

$\mathrm{Zu}$ diesem Verfahren habe ich noch folgendes $\mathrm{zu}$ bemerken. Das reine, trockene Taurocholat ist verhältnismäßig schwerlöslich in Alkohol, namentlich in absolutem. Es löst sich allerdings besser in warmem Alkohol; beim Abkühlen fällt es aber zum Teil wieder aus als eine grobflockige oder tonerdehydratähnliche Fällung, wenn nicht die ganze Lösung fast gallertähnlich erstarrt. Dies erschwert bisweilen, namentlich bei Verwendung von Rindergalle, die Darstellung der Säure; es macht auch eine ziemlich große Menge Alkohol not- 
wendig, und dem entsprechend wird sowohl der Ätherverbrauch, wie der unvermeidliche Verlust an in Lösung gebliebener Taurocholsäure größer. Um diesen Übelstand zu vermeiden, habe ich folgende Abänderung gemacht, die, soweit ich bisher gesehen habe, zu guten Resultaten führt. Das reine, fein zerriebene Taurocholat wird mit Alkohol, welcher $2-3 \%$ Säure enthält, bei Zimmertemperatur verrieben und in einem kleinen Kolben damit umgeschüttelt. Es findet auch hier eine Zersetzung statt. Die Taurocholsäure wird von dem säurehaltigen Alkohol gelöst und es bleibt Sulfat bezw. Chlorid zurück. Die abfiltrierte saure, alkoholische Lösung wird mit Äther gefällt.

Wenn die alkoholische Lösung der freien Taurocholsäure zufälligerweise etwas $\mathrm{zu}$ viel Wasser enthalten würde, kann es sich ereignen, daß man nach Zusatz von Äther die Taurocholsäure in Wasser gelöst als eine untere Schicht erhält. Wenn man diese von der Ätherlösung getrennte Schicht mit einer passenden Menge absoluten Alkohols mischt, kann man die Säure mit Äther leicht ausfällen.

Hinsichtlich der zu verwendeten Mineralsäure gebe ich aus theoretischen Gründen der Schwefelsäure den Vorzug, weil die Entfernung der Sulfate mit Alkohol leichter und sicherer als die der Chloride gelingt. $\mathrm{Zu}$ meinen allermeisten und insbesondere $\mathrm{zu}$ den in dem folgenden mitgeteilten Versuchen habe ich indessen immer nur Chlorwasserstoffsäure verwendet. Es war nämlich immer notwendig, Schwefelbestimmungen auszuführen, und aus dem Grunde mußte jede Verunreinigung mit Schwefelsäure oder Sulfaten vollständig ausgeschlossen werden können.

Die nach der neuen Methode aus verschiedenen Gallen dargestellte Taurocholsäure hatte immer dieselben Eigenschaften. Sie hatte immer einen süßen, nur wenig bitteren, an Süßholzextrakt erinnernden Geschmack. Sie kristallisierte bei rascher Kristallisation in Gruppen von feinen Nadeln; bei mehr langsamer Kristallisation in schönen Prismen, meistens mit durch zwei Flächen abgeschnittenen Enden. Sie löste sich leicht in Wasser mit stark saurer Reaktion, die kristallisierte Säure jedoch viel weniger leicht als die amorphe. Sie löste sich 
auch in Alkohol, die kristallisierte jedoch nicht besonders leicht. In Äther, Benzol und Aceton löste sie sich nicht. Die Säure konnte tagelang in offenem Gefäße an der Luft stehen, ohne durch Wasseraufnahme zu zerfließen. Dagegen konnte sie nicht über $100^{\circ}$ C. ohne Zersetzung erhitzt werden. Selbst beim Erhitzen auf dem Wasserbade fand unter gelbbrauner Färbung eine Zersetzung der Säure statt. Da ich bisher nur meine Bemühungen auf die Ausarbeitung einer Methode zur Reingewinnung der Säure gerichtet habe, kann ich jedoch hier keine weiteren Angaben über die Eigenschaften der letzteren geben.

Die Darstellung der Taurocholsäure ist also nach dem obigen Verfahren eine sehr leichte und einfache Aufgabe, vorausgesetzt nur, daß man über reines Taurocholat verfügen kann. Gerade in der Reindarstellung dieses Salzes liegt aber die Schwierigkeit, und in dieser Hinsicht verhalten sich die Gallen verschiedener Tiere etwas verschieden. Bisher habe ich nur mit drei verschiedenen Arten Galle, nämlich Dorsch-, Rinder- und Hundegalle, gearbeitet, und da ich nun einige Belege für die Brauchbarkeit der obigen Methode mitteile, muß ich für jede Art Galle über die Darstellung des Taurocholates und der freien Säure in einem Zusammenhange berichten.

Darstellung der Taurocholsäure aus Dorschgalle.

Aus dieser Galle gelingt die Darstellung der Säure am leichtesten. Die Galle wird erst mit Eisenchlorid gefällt. Die von dem stark gefärbten, nicht besonders reichlichen Niederschlage abfiltrierte Flüssigkeit wird nach Tengströms Verfahren verarbeitet. Sie wird also zur Ausfällung von überschüssigem Eisen mit $\mathrm{Na}_{2} \mathrm{CO}_{3}$-Lösung alkalisch gemacht, worauf das neue Filtrat fast neutralisiert wird. Durch Sättigung mit $\mathrm{NaCl}$ wird nun das Taurocholat (fast rein weiß) ausgefällt, mit gesättigter NaCl-Lösung ausgewaschen, noch einmal aus wässeriger Lösung mit NaCl gefällt und durch wiederholtes Eintrocknen und Auflösen in Alkohol von NaCl befre . Das Taurocholat ist rein weiß, kann aus alkoholischer Lösung mit Äther kristallisiert erhalten werden und hat den geforderten Schwefelgehalt, $5,9-5,95 \%$. 
Die freie Säure wurde nach dem oben angegebenen Verfahren leicht gewonnen. Sie kristallisierte leicht und rasch und wurde im ganzen dreimal umkristallisiert. Je nach der rascheren oder langsameren Kristallisation bestand sie aus feinen Nadeln oder vierseitigen Prismen, deren Enden von zwei Flächen schief abgeschnitten waren.

Die zur Schwefelbestimmung verwendete kristallisierte Säure erwies sich als fast absolut chlorfrei. $0,3 \mathrm{~g}$ lieferten eine kaum sicher wägbare Menge Asche $(0,0002 \mathrm{~g}=0,06 \%)$ und die Säure war also fast aschefrei. Sie konnte tagelang in offenem Gefälle ohne sichtbare Veränderung stehen und wurde im Exsikkator leicht von konstantem Gewicht erhalten. Die Schwefelbestimmung ergab folgendes:

$$
\begin{gathered}
0,467 \mathrm{~g} \text { Substanz lieferten } 0,204 \mathrm{~g} \mathrm{BaSO}_{4} \\
=0,028 \mathrm{~g} \text { Schwefel }=6,0 \% \mathrm{~S} .
\end{gathered}
$$

Der Gehalt an Schwefel war also $=6,0 \%$, während die Formel der wasserfreien Säure 6,21\% S erfordert. Da ich die Säure nie aus wasserfreiem, sondern nur aus wasserhaltigem Alkohol durch Ätherzusatz in Kristallen erhielt, liegt die Annahme nahe, daß die Säure Kristallwasser enthält. Inwieweit dies der Fall ist, kann ich jedoch noch nicht sicher angeben. Jedenfalls kann das Kristallwasser (resp. Kristallalkohol) nicht durch das Vacuum allein entfernt werden, denn das Gewicht bleibt wochenlang im Exsikkator unverändert. Beim Erhitzen auf $100^{\circ} \mathrm{C}$. und sogar bei etwas niedrigerer Temperatur zersetzt sich die Säure unter Gelb- oder Braunfärbung. Beim Erhitzen der im Exsikkator getrockneten Säure auf $90^{\circ}$ kürzere Zeit habe ich jedoch einen Gewichtsverlust, welcher $2-30 \%$ Wasser entspricht, beobachtet, ohne daß hierbei eine merkbare Veränderung der Säure stattfand. Ich habe diese Frage bisher nicht weiter verfolgt, erlaube mir aber zu bemerken, daß eine Säure von der Formel $\mathrm{C}_{26} \mathrm{H}_{40} \mathrm{NSO}_{7}+\mathrm{H}_{2} \mathrm{O}$ gerade den gefundenen Wert $6,0 \%$ Schwefel enthalten würde.

Die aus Dorschgalle dargestellte Taurocholsäure habe ich ein paarmal zur Gewinnung der entsprechenden Cholalsäure mit 10\% iger Natronlauge 8-9 Stunden im Autoklaven bei ein wenig über $100^{\circ} \mathrm{C}$. erhitzt. Die Cholalsäure kristallisierte leicht 
in der für gewöhnliche Cholsäure typischen Kristallform, sie gab sehr schön die Myliussche Jodreaktion und hatte den Schmelzpunkt $195^{\circ} \mathrm{C}$. Sie war also gewöhnliche Cholsäure und die entsprechende gepaarte Säure also gewöhnliche Taurocholsäure.

$\mathrm{Da}$ das Taurocholat und die freie Säure bezüglich des Schwefelgehaltes nur wenig voneinander abweichen, beweist der oben gefundene Wert, 6,0\% Schwefel, eigentlich nur, daß eine in Betracht kommende Verunreinigung mit Cholsäure (oder Glykocholsäure) ausgeschlossen war. Dagegen konnte fast ebensogut ein Taurocholat vorliegen, wenn man nur den Schwefelgehalt in Betracht zieht. Da aber das Taurocholat bei der Verbrennung eine reichliche Menge Asche, jedenfalls nie weniger als $13 \%$ (als $\mathrm{Na}_{2} \mathrm{SO}_{4}$ berechnet) gibt, während das analysierte Präparat nur $0,06 \%$ Asche lieferte, kann kein Zweifel darüber bestehen, daß die Darstellung kristallisierter Taurocholsäure hier gelungen war.

\section{Darstellung der Taurocholsäure aus Rindergalle.}

Die Reingewinnung des Taurocholates aus der Rindergalle ist mit großen Schwierigkeiten verknüpft, und darum eignet sich auch diese Galle, wenigstens so lange die Methode nicht besser ausgearbeitet worden ist, nicht gut zur Darstellung der Taurocholsäure. Die zur Darstellung des Salzes befolgte Methode war im Prinzipe die von Tengström ausgearbeitete.

Die durch $\mathrm{NaCl}$ nicht direkt aussalzbare Galle wurde also erst mit Bleizucker und dann wiederholt mit Eisenchlorid gefällt, bis die Galle durch $\mathrm{NaCl}$ gefällt werden konnte. Nach diesem Verfahren erhält man sowohl aus der Rindergalle wie aus anderen Gallen ohne vorgängige Entfärbung die ausgesalzenen Gallensalze fast sogleich rein weiß. Bei der weiteren Verarbeitung dieser Gallensalze wich ich insofern von Tengström $\mathrm{ab}$, als ich nicht mit $\mathrm{NaCl}$, sondern mit $\mathrm{KCl}$ fällte. Dies geschah aus dem Grunde, daß nach Tengström das letztgenannte Salı nur das Taurocholat, nicht aber das Glykocholat fällen soll.

Zur Ausfällung des Taurocholates löste ich das $\mathrm{KCl}$ (etwa $34 \mathrm{~g}$ auf je $100 \mathrm{ccm}$ Lösung) in der Gallensalzlösung unter Erwärmen bis fast zum Sieden, filtrierte heiß und ließ erkalten. 
Beim Erkalten schied sich das Taurocholat (nebst etwas $\mathrm{KCl}$ ) grobflockig aus. Die Ausscheidung geschieht aber nicht auf einmal, denn wenn man die Fällung abfiltriert, treten im Filtrate, bisweilen erst am folgenden Tage, neue Ausscheidungen auf, die das Aussehen von großen lockeren Fibringerinnseln oder von Schimmelbildungen haben. Sämtliche Ausscheidungen wurden gesammelt, ausgepreßt, in Wasser gelöst, noch einmal mit $\mathrm{KCl}$ gefällt, ausgepreßt, in Alkohol gelöst und durch Filtration von $\mathrm{KCl}$ getrennt. Durch wiederholte Alkoholbehandlung in der gewöhnlichen Weise wurde das Salz gereinigt.

Das in dieser Weise gewonnene Salz enthält, selbst wenn es keine nennenswerte Chlorreaktion gibt, sowohl Kalium als Natrium in, wie es scheint, wechselnden Mengen. Da das Natriumsalz der Taurocholsäure 5,96 und das Kaliumsalz 5,79\% Schwefel enthält, ist es, ohne gleichzeitige Bestimmung des Kalium- und Natriumgehaltes, nicht möglich, aus der Bestimmung des Schwefelgehaltes allein sichere Aufschlüsse über die Reinheit des Taurocholates zu gewinnen. Da ich aber Werte für den Schwefelgehalt gefunden habe, die, nach gebührender Korrektion für die verunreinigenden Chloride, in verschiedenen Präparaten zwischen 5,38 und 5,79\% $\mathrm{S}$ schwankten, ist es offenbar, daß die Reindarstellung des Taurocholates aus der Rindergalle mir nicht gelungen ist. Tengström gelangte zu besserem Resultat, indem er nämlich durch Aussalzen mit $\mathrm{NaCl}$ ein Präparat mit $5,93 \% \mathrm{~S}$, d. h. etwa $99 \%$ Taurocholat erhielt.

Das Aussalzen mit $\mathrm{KCl}$ führte also nicht zu besseren Resultaten als die Anwendung von $\mathrm{NaCl}$, welches Salz Präparate von ebenso hohem Schwefelgehalt wie die mit $\mathrm{KCl}$ dargestellten Präparate lieferte. Die Anwendung des letztgenannten Salzes scheint also keine besonderen Vorteile darzubieten, eher das Gegenteil. Die Fällung tritt sehr schleppend auf, und in verdünnten Lösungen findet bisweilen in den ersten 24 Stunden überhaupt keine Ausscheidung statt. Erst später treten nach und nach die eigentümlichen faserigen und lockeren Niederschläge auf, und ein bedeutender Rest bleibt in Lösung. Es scheint mir übrigens etwas zweifelhaft zu sein, ob nicht etwa vorhandenes Glykocholat zum Teil mit niedergerissen wird. 
Wenn nämlich, wie Tengström fand, das Glykocholat in reinem Zustande von $\mathrm{KCl}$ nicht gefällt wird, so schließt dies doch nicht aus, daß aus einem Gemenge der zwei Salze Doppelverbindungen derselben durch das $\mathrm{KCl}$ ausgefällt werden könnten. Es sprechen nämlich mehrere Beobachtungen für die Existenz derartiger Doppelverbindungen, wenn ich auch noch nicht auf diese Frage habe näher eingehen können.

Die von Tengström ausgearbeitete Methode für die Reindarstellung des Taurocholates aus Rindergalle ist allerdings als ein wesentlicher Fortschritt anzusehen; ich glaube jedoch nicht, daß man nach dieser Methode immer ein reines Präparat gewinnen kann. Mir ist dies wenigstens nicht gut gelungen. Da die Taurocholeinsäure noch nicht in reinem Zustande dargestellt worden ist, und da man folglich noch nicht die Fällbarkeit des Taurocholeinates kennt, darf man übrigens die Möglichkeit nicht außer acht lassen, daß das nach der obigen Methode isolierte Taurocholat von dem Salze der Taurocholeinsäure verunreinigt sei. Die Methode zur Reingewinnung des Taurocholates aus Rindergalle muß also noch weiter ausgearbeitet werden.

Das Taurocholat ist, wie in dem vorigen bemerkt wurde, nicht leicht löslich in kaltem Alkohol. Das Taurocholat aus Rindergalle scheint besonders ziemlich schwerlöslich zu sein, was vielleicht durch irgend eine Beimengung bedingt ist. Aus dem Grunde habe ich auch bei der Darstellung der freien Säure aus dem Taurocholate der Rindergalle die obige Abänderung des Verfahrens, d. h. das Zerreiben des Salzes mit säurehaltigem Alkohol, benutzt. Die Methode war im übrigen dieselbe und dürfte also keiner ausführlicheren Erwähnung bedürftig sein. Die Säure, welche zwei- oder dreimal umkristallisiert wurde, hatte dieselben Eigenschaften und dieselbe Kristallform wie die Säure aus Dorschgalle. Sie gab ganz typische gewöhnliche Cholsäure, und es konnte aus der Rohcholalsäure keine Choleinsäure gewonnen werden. Die Säure war fast ganz frei von Mineralstoffen, der Gehalt an Schwefel in den verschiedenen Präparaten schwankte aber zwischen 5,80 und 5,910\%. Der letztgenannte Wert stimmt allerdings hinreichend gut zu einer Säure von der Formel $\mathrm{C}_{26} \mathrm{H}_{45} \mathrm{NSO}_{7}+\mathrm{H}_{2} \mathrm{O}$ (=6,0\% Schwefel); 
die übrigen Zahlen sind aber alle zu niedrig und der Wert 5,80\% $\mathrm{S}$ würde einem Präparate mit einem Gehalte von nur 96,70\% einer solchen Säure entsprechen. Aus der Rindergalle ist also die Reindarstellung sowohl der freien Säure wie des Taurocholates mir noch nicht gut gelungen, und die Methode ist einer weiteren Vervollkommnung bedürftig.

Darstellung der Taurocholsäuren aus Hundegalle.

Als Material zur Darstellung der Taurocholsäure ist allgemein die Hundegalle empfohlen worden. Dies ist aber nur unter der Voraussetzung zu billigen, daß die Hundegalle nur gewöhnliches Taurocholat enthält. Wem aber der süße, nur sehr schwach bittere Geschmack des gewöhnlichen Taurocholates bekannt ist, der wird es sehr wahrscheinlich finden, daß die Hundegalle neben der Taurocholsäure auch eine andere, mehr bitterschmeckende Gallensäure enthält. Dies war auch einer der Gründe, warum ich zu einer Untersuchung der Hundegalle ging.

Die Hundegalle wurde unmittelbar nach dem Töten des Tieres in überschüssigen Alkohol gegossen und in dieser Weise aufbewahrt, bis von mehreren Hunden eine für die Untersuchung genügende Menge Galle aufgesammelt worden war. Die von «Schleim * abfiltrierte alkoholische Lösung wurde auf dem Wasserbade eingetrocknet, der Rückstand in starkem Alkohol gelöst und diese Lösung auf einmal mit viel Äther unter Umschütteln versetzt. In dieser Weise erhält man die gallensauren Salze nicht als zähe Klumpen und Massen, sondern als eine nicht stark gefärbte, flockige Fällung, während die Hauptmasse des Farbstoffes in der alkoholisch-ätherischen Lösung bleibt. Die abfiltrierten und ausgepreßten gallensauren Salze wurden in Wasser gelöst und weiter verarbeitet.

Diese Lösung wurde fraktioniert mit Eisenchlorid gefällt. Die ersten Niederschläge, welche ein teerartiges Aussehen hatten und fast allen Farbstoff aufnahmen, wurden gesondert verarbeitet. Sie wurden mit Sodalösung zersetzt, die Lösung eingetrocknet und der Rückstand mit warmem Alkohol extrahiert, wobei, wie gewöhnlich, der ungelöste Rest reichliche Mengen umgewandelten Farbstoffs zurückhielt. Die abfiltrierte alkoholische 
Lösung wurde eingetrocknet und der Rückstand mit kaltem absoluten Alkohol behandelt. Hierbei löste sich ein großer Teil, während ein anderer, aus Flocken und Schollen bestehender, fast weißer Teil (aus Seifen bestehend) zurückblieb. Das Eintrocknen des Filtrates und Wiederauflösen in kleinen Mengen kalten Alkohols wurden wiederholt, bis alles in Alkohol leicht und klar löslich war. Diese Lösung hatte einen ekelhaft bitteren Geschmack, welcher schon an und für sich zeigte, daß hier eine ganz andere Gallensäure als die gewöhnliche Taurocholsäure vorlag. Diese, aus der Eisenfraktion 1 erhaltene Lösung wurde mit der aus der Eisenfraktion 2 gewonnenen vereinigt.

Das Filtrat von der Eisenfraktion 1 wurde wiederholt mit Eisenchlorid, unter Abstumpfung der sauren Reaktion mit Soda und Verdünnen mit Wasser, gefällt. Die Hundegalle verhielt sich hierbei in derselben Weise wie die Rindergalle. Wenn man die Lösung mit Eisenchlorid versetzt, kommt man nämlich bald zu einem Punkte, wo weiterer Zusatz von Eisenchlorid keine Fällung erzeugt. Versetzt man nun das eisenreiche, sauer reagierende Filtrat mit Soda bis zu schwach saurer Reaktion, so tritt ein neuer, blaß gelbbrauner Niederschlag auf. Zusatz von Eisensalz zu dem neuen Filtrate erzeugt vielleicht eine neue Fällung; wenn dies aber nicht der Fall ist, kann man regelmä6ig durch Verdünnung mit Wasser einen neuen Niederschlag erhalten. Setzt man dem Filtrate auf einmal eine größere Menge Eisenchlorid hinzu, so bleibt die Lösung oft ganz klar, stumpft man aber die saure Reaktion größtenteils mit Soda $a b$, so erhält man wiederum einen blaß gelbbraunen Niederschlag, welcher Gallensäuren enthält. ${ }^{j}$ ) Ist die Lösung infolge der allmählich stattgefundenen Verdünnung durch Eisenchlorid nicht mehr fällbar, so kann man durch Sodazusatz, Eintrocknen und Alkoholbehandlung die gallensauren Salze isolieren und in Wasser lösen, und man kann nun wieder eine Eisenfällung erhalten. In dieser Weise kommt man bei der Fällung der Galle mit Eisenchlorid fast nie ganz zu Ende, wenn auch die Fällungen zuletzt nur sehr geringfügig sind.

1) Dieser Niederschlag löst sich leicht in Alkohol und unterscheidet sich sowohl hierdurch, wie durch die Farbe von Eisenoxydhydrat. 
Da auch die Hundegalle in der nun geschilderten Weise bei der Fällung mit Eisenchlorid sich verhielt, verfuhr ich so, daß ich so lange mit Eisenchlorid fällte, bis die Eisenniederschläge, mit Soda zersetzt, eine stark süß, nur wenig bitter schmeckende Lösung gaben. Das Endfiltrat von den Eisenfällungen, als Fraktion 4 bezeichnet, wurde auf gewöhnliche Taurocholsäure verarbeitet.

Von den Eisenfällungen wurden diejenigen, welche eine stark süß schmeckende Lösung gaben und welche dementsprechend hauptsächlich Taurocholat enthielten, als Fraktion 3 aufbewahrt. Ihre Menge war so klein, daß eine weitere Verarbeitung nicht unternommen wurde. Die ersten Eisenfällungen dagegen, welche I,ösungen von stark bitterem Geschmack lieferten, wurden als Fraktion 2 gesammelt und auf eine etwa vorhandene zweite gepaarte Säure verarbeitet.

$\mathrm{Zu}$ dem Ende wurden sie mit Sodalösung zersetzt, das Filtrat eingetrocknet und der Rückstand mit Alkohol behandelt. Diese Lösung, welche einen viel stärker bitteren Geschmack als gewöhnliches Taurocholat hatte, wurde mit der obigen, aus Fraktion 1 erhaltenen Lösung vereinigt und eingetrocknet. Der Rückstand wurde in wenig Alkohol gelöst, die Lösung filtriert, mit salzsäurehaltigem Alkohol versetzt und nach dem Filtrieren mit Äther gefällt. Die ausgefällte amorphe Säure wurde in Alkohol gelöst und nach Zusatz von ein wenig Wasser mit Äther versetzt. Sie schied sich auch jetzt amorph aus, ging aber nach längerer Zeit größtenteils in Ballen von feinen Nadeln über.

$\mathrm{Da}$ die geringe Menge der Säure nicht zu allen den erwünschten Proben ausreichend war, und da es vor allem darauf ankam, zu wissen, ob eine andere Taurocholsäure als die gewöhnliche hier vorlag, zersetzte ich die Säure durch Sieden mit Natronlauge von $8 \%$ im Autoklaven bei etwa $100^{\circ} \mathrm{C}$. Aus der Lösung wurde die Cholalsäure mit Salzsäure gefällt und in dem Filtrate konnte ich nach dem von mir angegebenen Verfahren ${ }^{1}$ ) leicht Taurin nachweisen, jedoch in so kleiner Menge, daß ich eine Bestimmung des Schwefelgehaltes nicht ausführen konnte.

1) Diese Zeitschrift, Bd. XXXII, S. 456. 
Die ausgewaschene und getrocknete Cholalsäure gab nur eine äußerst schwache Jodreaktion. Um sie weiter zu reinigen, führte ich sie in das Natriumsalz über. Die wässerige Lösung dieses Salzes, welche einen intensiv bitteren Geschmack hatte, wurde mit $\mathrm{BaCl}_{2}$-Lösung versetzt. Es entstand hierbei eine starke feinkörnige Fällung, die erst langsam zu Boden sich setzte und dort eine zähe, honigähnliche Schicht bildete. Das in Alkohol leicht lösliche Baryumsalz wurde in das Natriumsalz wieder übergeführt und die Lösung der letzteren in Wasser mit Salzsäure gefällt.

Die so gefällte, rein weiße, in Wasser sehr schwerlösliche Cholalsäure wurde in ein wenig siedendem absoluten Alkohol gelöst und am folgenden Tage hatten helle farblose Kristalle sich ausgeschieden. Auch aus der Lösung des Alkalisalzes in Wasser erhielt ich die Säure nach Zufügung von Äther und Salzsäurezusatz in Nadeln und zum Teil in 6 seitigen Tafeln. Die so gewonnene Cholalsäure gab keine Spur einer Jodreaktion und der Schmelzpunkt war $182^{\circ}$ C. Hiermit war es also bewiesen, daß die untersuchte Hundegalle eine Taurocholsäure enthielt, welche nicht Cholsäure, sondern eine andere Cholalsäure gab. Ob diese gepaarte Säure Taurocholeinsäure ist, habe ich noch nicht ermitteln können und zwar wegen Mangels an Material. Es ist leider hier in Upsala sehr schwer, Hundegalle $\mathrm{zu}$ bekommen, und bisher habe ich leider nicht mehr als $100 \mathrm{ccm}$ frische Galle in Arbeit nehmen können. Die erhaltene Cholalsäure ähnelt jedoch sehr der Choleinsäure und ist vielleicht mit ihr identisch.

Aus dem Endfiltrate (Fraktion 4), welches auf gewöhnliche Taurocholsäure $\mathrm{zu}$ verarbeiten war, wurde mit $\mathrm{Na}_{2} \mathrm{CO}_{3}$ im Überschuß das Eisen entfernt. Das Filtrat wurde fast neutralisiert, eingetrocknet, der Rückstand mit Alkohol erschöpft und das Taurocholat durch wiederholtes Auflösen in Alkohol und Eintrocknen gereinigt. Die freie Säure wurde dann in derselben Weise wie aus der Dorschgalle dargestellt und 2 mal aus Alkohol durch Ätherzusatz umkristallisiert. Die Säure hatte dieselbe Kristallform wie die aus Dorsch- oder Rindergalle. Sie war nicht zerfließlich an der Luft und konnte nicht ohne 
Zersetzung auf $100^{\circ}$ G. erhitzt werden. Die Schwefelbestimmung ergab folgendes:

$0,369 \mathrm{~g}$ Substanz lieferten $0,161 \mathrm{~g} \mathrm{BaSO}_{4}$ $=0,02213 \mathrm{~g}$ Schwefel $=5,995$ oder rund $6,00 \% \mathrm{~S}$.

Der Schwefelgehalt, $6,0 \%$, war also derselbe wie in der Säure aus Dorschgalle und entspricht der Formel

$$
\mathrm{C}_{26} \mathrm{H}_{45} \mathrm{NSO}_{7}+\mathrm{H}_{2} \mathrm{O} \text {. }
$$

Der Rest dieser Säure wurde zur Darstellung der Cholalsäure mit $8 \%$ iger Lauge gekocht. Die Cholalsäure gab intensive Jodreaktion, hatte die typische Kristallform der Cholsäure und den Schmelzpunkt $195^{\circ}$ C. Die untersuchte gepaarte Säure war also gewöhnliche Taurocholsäure.

Die Brauchbarkeit der neuen Methode auch für die Hundegalle dürfte hiermit bewiesen sein. Die Schwierigkeit liegt auch hier in der Darstellung des Taurocholates, und zwar infolge der Anwesenheit von zwei Taurocholsäuren. Da ich bisher nur $100 \mathrm{ccm}$ Hundegalle verarbeitet habe, wage ich natürlich nicht, zu behaupten, daß die Hundegalle regelmäßig zwei gepaarte Säuren enthält, wenn ich auch dies sehr wahrscheinlich finde. Die von mir untersuchte Galle war nämlich gemischte Galle, die von 4 verschiedenen Individuen stammte. 